\title{
Ecosistemas digitales y su manifestación en el aprendizaje: Análisis de la literatura
}

\section{Digital ecosystems and their manifestation in learning: Analysis of literature}

\author{
Claudia Islas Torres \\ Universidad de Guadalajara. Guadalajara, México \\ cislas@cualtos.udg.mx \\ María del Rocío Carranza Alcántar \\ Universidad de Guadalajara. Guadalajara, México \\ mcarranza@ cualtos.udg.mx
}

\begin{abstract}
Resumen
A partir de que los medios digitales pueden considerarse como entes con identidad y vida propia que cambian en función de cómo se consumen y distribuyen los contenidos, se conciben como ecosistemas con sentido de evolución, muerte u obsolescencia; así, estos medios se hacen presentes en los procesos de aprendizaje, por lo que el objetivo de éste escrito es contribuir al estado del conocimiento sobre los ecosistemas digitales y su manifestación en el aprendizaje de los individuos, desde una postura analítica. El método para desarrollar dicho trabajo fue documental en sus fases heurística y hermenéutica, el criterio de selección basado en artículos científicos escritos en su mayoría en español. Los descriptores para la búsqueda fueron: ecosistemas digitales, aprendizaje y ecosistemas así como sociedad del conocimiento. Lo encontrado indica que la investigación en este ámbito aún es insuficiente, en su mayoría la literatura hallada habla de lo que debieran ser, cómo constituirse o las características de los ecosistemas asociados al aprendizaje, sin embargo, carecen de sustento empírico, de datos cuantitativos o cualitativos que comprueben lo alejado o cercano que está el discurso de la realidad que se vive tras la ubicuidad de las TIC y su presencia en los contextos formativos.
\end{abstract}

Palabras clave

Estado del conocimiento, ecosistemas digitales, aprendizaje.

\begin{abstract}
Since the digital media can be considered as objects with identity and life of their own that change depending on how the contents are consumed and distributed, those are conceived as ecosystems with a sense of evolution, death or obsolescence; thus, these means are present in the learning processes, so the purpose of this writing is to contribute to the state of knowledge about digital ecosystems and their manifestation in the learning of individuals, from an analytical standpoint. The method to develop this work was a documentary in its heuristic and hermeneutic phases, the criterion of selection based on scientific articles written mostly in Spanish. The descriptors for the search were: digital ecosystems, learning, and ecosystems as well as a knowledge society. This finding indicates that research in this area is still insufficient, most of the literature found speaks of what those should be, how to constitute themselves or the characteristics of the ecosystems associated with learning, however, lack empirical support, quantitative or qualitative data that prove how far or near the discourse of the reality that is lived after the ubiquity of the ICT and its presence in the formative contexts.
\end{abstract}

Key words

State of knowledge, digital ecosystems, learning 


\section{Introducción}

En los últimos años los dispositivos tecnológicos e internet con sus aplicaciones y herramientas han tenido alto impacto en distintos ámbitos entre ellos el educativo, por lo que surge el interés de presentar este acercamiento al estado del conocimiento respecto a los ecosistemas digitales y su manifestación en el aprendizaje. La inquietud se respalda en la observación de las tendencias relacionadas al uso de herramientas y aplicaciones tecnológicas en los entornos y procesos de formación, además; en la intención de comprender las formas en que sus usos trascienden los horarios comunes, los espacios, cómo se transforman las interacciones y la comunicación, y cómo las aplicaciones parecen convertirse en un espacio alternativo donde se posibilita la producción y socialización del conocimiento, además del aprendizaje para la vida.

En este sentido, se reconoce que existe gran auge en el uso de las redes sociales, los blogs, la curación de contenidos, las plataformas educativas, los MOOC (cursos en línea masivos y abiertos por sus siglas en inglés), las aplicaciones especializadas, entre otros; opciones que posibilitan la transmisión de información desde varias fuentes, en distintos formatos y soportes que además son de acceso instantáneo, configurando así servicios que las personas pueden aprovechar para adaptarse o des-adaptarse tal como lo harían en un ecosistema natural.

Lo anterior, coincide con la postura de García-Peñalvo (2016) quien afirma que la influencia que tienen las tecnologías en el quehacer cotidiano de todas las personas, provoca una transferencia consciente o no hacia los contextos en que se desenvuelven y por ende, en la forma en que gestionan el conocimiento tanto a nivel individual como social.

La irrupción de las Tecnologías de la Información y la Comunicación (TIC) significan un punto de inflexión conceptual y metodológico en la forma en que tanto instituciones educativas como otro tipo de organizaciones afrontan los procesos de formación y la gestión del conocimiento, por tanto, estas herramientas dejan de ser el centro de atención y se convierten en parte de un ecosistema digital, donde su presencia se hace transparente y se incluye en el accionar cotidiano de quienes están en él gestionando el aprendizaje, ya sea de forma individual o colectiva (García-Peñalvo \& Seoane-Pardo, 2015).

Es así, que las tecnologías que median la educación con las ventajas y problemas que implican trascienden del contexto académico y se insertan en cualquier institución para convertirse en herramientas de gestión del conocimiento digital y del capital humano de la misma, poniendo de manifiesto que las tecnologías de la información y las comunicaciones provocan una ruptura de las fronteras clásicas entre distintos tipos de aprendizaje tales como el formal, no formal e informal, dando lugar a la construcción del conocimiento y la interacción social a través de la ubicuidad de las TIC (Cabero, 2015; Sevillano \& Vázquez, 2013; Attwell, 2013).

En este sentido, los ecosistemas de aprendizaje abren posibilidades inmensas para que cada aprendiz pueda construir su trayectoria de aprendizaje a partir de sus necesidades o intereses; sin embargo, también implica riesgos para quienes sus condiciones de vida 
limitan fuertemente las oportunidades, experiencias y recursos de aprendizaje a su alcance (Coll, 2016).

Lo anterior implica complejidad para entender las TIC y su alta penetración en todos los ámbitos, por lo que se requiere de abordajes integrales que lleven a la comprensión de los problemas, desafíos, desarrollo, ejecución y gestión de estrategias que permitan conocer la evolución que emerge del uso, apropiación y explotación de las tecnologías en un entramado de relaciones e interacciones que se producen, evolucionan y mueren.

En este sentido, el presente escrito tiene por objetivo contribuir al estado del conocimiento sobre los ecosistemas digitales y su manifestación en el aprendizaje de los individuos, desde una postura analítica. El método para desarrollar dicho trabajo fue documental en sus fases heurística y hermenéutica y el criterio de selección se basó en artículos científicos escritos en su mayoría en español. Los descriptores para la búsqueda fueron: ecosistemas digitales, aprendizaje y ecosistemas y sociedad del conocimiento.

\section{Metodología}

\subsection{Proceso de la investigación}

Para lograr el objetivo de este trabajo se procedió a la búsqueda, recuperación y análisis de información primaria, derivada de la investigación y registro de diferentes autores que generalmente expusieron su conocimiento a través de artículos de tipo científico, publicados en revistas indexadas escritas en idioma español (fase heurística).

Por lo que respecta a la fase hermenéutica se analizaron, interpretaron y clasificaron los documentos recopilados y cuyo aporte refería a los ecosistemas digitales, aprendizaje y ecosistemas y sociedad del conocimiento. Como resultado de estas fases en la tabla 1 se presenta un concentrado de la literatura utilizada como fuente de análisis y que da cuerpo al resto del documento.

Tabla 1. Muestra de la selección de información.

\begin{tabular}{|c|c|c|c|}
\hline $\begin{array}{c}\text { Temática que se } \\
\text { aborda }\end{array}$ & Autor (es) & $\begin{array}{c}\text { Año de } \\
\text { publicación }\end{array}$ & Revista o Libro \\
\hline $\begin{array}{l}\text { Ecosistemas de formación y } \\
\text { competencia mediática: } \\
\text { Valoración internacional } \\
\text { sobre su implementación en } \\
\text { educación superior }\end{array}$ & $\begin{array}{l}\text { E. Álvarez-Arregui, } \\
\text { A. } \quad \text { Rodríguez } \\
\text { Martín, } \\
\text { Madrigal- } \\
\begin{array}{ll}\text { Maldonado, R. B. } \\
\text { Grossi-Sampedro }\end{array} \\
\end{array}$ & 2017 & Comunicar \\
\hline $\begin{array}{ll}\text { Entornos personales } & \text { de } \\
\text { aprendizaje }\end{array}$ & G. Attwell & 2013 & $\begin{array}{l}\text { Entornos personales de } \\
\text { aprendizaje: Claves para el } \\
\text { ecosistema educativo en Red }\end{array}$ \\
\hline $\begin{array}{llr}\text { La } & \text { sociedad } & \text { del } \\
\text { conocimiento y los entornos } \\
\text { digitales }\end{array}$ & C. Barroso-Jerez & 2013 & $\begin{array}{l}\text { Teoría de la educación. } \\
\text { Educación y cultura en la } \\
\text { sociedad de la Información }\end{array}$ \\
\hline $\begin{array}{l}\text { Reflexiones educativas sobe } \\
\text { las TIC }\end{array}$ & J. Cabero & 2015 & CEF \\
\hline $\begin{array}{l}\text { Los educadores de la era } \\
\text { digital }\end{array}$ & J. Cabero & 2017 & Notandum \\
\hline
\end{tabular}

Ecosistemas digitales y su manifestación en el aprendizaje: Análisis de la literatura. Claudia Islas Torres, María del Rocío Carranza Alcántar. 


\begin{tabular}{|c|c|c|c|}
\hline $\begin{array}{lll}\text { Sociabilidad virtual: } & \text { la } \\
\text { interacción social en } & \text { el } \\
\text { ecosistema digital } & & \\
\end{array}$ & $\begin{array}{l}\text { M.D. Cáceres, G. } \\
\text { Brándle, J. A Ruíz }\end{array}$ & 2017 & Historia y comunidad social \\
\hline $\begin{array}{l}\text { Los ecosistemas educativos } \\
\text { y el fracaso escolar }\end{array}$ & R. Choque & 2009 & $\begin{array}{l}\text { Revista Iberoamericana de } \\
\text { Educación }\end{array}$ \\
\hline $\begin{array}{l}\text { La educación formal en la } \\
\text { nueva ecología del } \\
\text { aprendizaje: } \\
\text { retos tendencias, } \\
\text { investigación }\end{array}$ & C. Coll & 2013 & $\begin{array}{l}\text { Aprendizaje y educación en la } \\
\text { sociedad digital }\end{array}$ \\
\hline $\begin{array}{ll}\text { Personalización } & \text { del } \\
\text { aprendizaje escolar } & \end{array}$ & C. Coll & 2016 & $\begin{array}{l}\text { El qué, el por qué, y el cómo } \\
\text { de un reto insoslayable }\end{array}$ \\
\hline $\begin{array}{l}\text { La universidad ante el reto } \\
\text { del aprendizaje ubicuo con } \\
\text { dispositivos móviles }\end{array}$ & $\begin{array}{l}\text { M. L García, E. } \\
\text { Vázquez }\end{array}$ & 2013 & EDETANIA \\
\hline $\begin{array}{lr}\begin{array}{l}\text { Análisis } \\
\text { conceptos, }\end{array} & \begin{array}{r}\text { diversos } \\
\text { b-learning, } \\
\text { innovación }\end{array} \\
\text { educativa, } \\
\text { ecosistemas tecnológicos }\end{array}$ & F. García Peñalvo & $\begin{array}{c}2013, \\
2015,2015\end{array}$ & $\begin{array}{l}\text { EKS, Congresos sobre } \\
\text { educación }\end{array}$ \\
\hline $\begin{array}{l}\text { Niños nativos digitales en la } \\
\text { sociedad del conocimiento: } \\
\text { acercamientos conceptuales } \\
\text { a sus competencias }\end{array}$ & M. A. Ibarra & 2010 & Razón y palabra \\
\hline $\begin{array}{l}\text { El futuro de la educación } \\
\text { superior }\end{array}$ & D. Reig & 2010 & REIRE \\
\hline $\begin{array}{l}\text { La universidad ante el reto } \\
\text { del aprendizaje ubicuo }\end{array}$ & $\begin{array}{l}\text { M. L Sevillano, E. } \\
\text { Vázquez }\end{array}$ & 2013 & EDETNIA \\
\hline
\end{tabular}

En los siguientes apartados se expone la información recuperada de estas fuentes así como de otros referentes que sirvieron como fundamento para dicho análisis.

\section{Conceptualizando los ecosistemas digitales}

Iniciamos esta conceptualización a partir de la premisa de que el aprendizaje se produce y producirá cada vez más a lo ancho de la vida, es decir; en una variedad de contextos de actividad y en todos los periodos vitales de los individuos, apoyándose en gran medida en las TIC como herramientas que posibilitan espacios de formación que están dentro o fuera de las instituciones de educación formal. En este sentido, también se involucran nuevos nichos de aprendizaje reforzando contextos tradicionales de actividad y desarrollo, tales como la familia, comunidad, instituciones culturales y de ocio o trabajo, etcétera (Coll, 2016).

A decir de García-Peñalvo (2016) los ecosistemas digitales suponen una mejora respecto a los sistemas de información tradicionales, puesto que el abanico de posibilidades de éstos permite cubrir cualquier tipo de necesidad que surja en una entidad, gracias a su estructura modular, a la importancia que se le da a los flujos de información establecidos entre los módulos y a la base metodológica que debe sustentar todo ecosistema.

Este concepto se ha tomado de la biología y utilizado desde hace un tiempo para describir los sistemas de software adaptativo, los ambientes de producción, reutilización y adaptación de contenidos, que se someten a un ciclo de retroalimentación mantenido durante cierto tiempo, produciendo evolución en las especies y el contexto desde los 
flujos de innovación-aceptación-consolidación-obsolecencia (Reyna, 2011; Motz \& Rodés, 2013; García-Peñalvo, 2016).

Pero, cuál es el sustento del concepto ecosistema digital, a decir de Motz \& Rodés (2013); Reyna (2011) y Choque (2009), éste se basa en la metáfora de los sistemas de biología, donde se denomina ecosistema al conjunto de elementos bióticos (seres vivos) y abióticos (suelo, agua, luz, minerales, etcétera) que están relacionados interactuando entre sí. Relaciones que se producen en un lugar determinado y delimitado del resto del entorno, capaz de asimilar materia y energía que se produce para que las distintas especies de seres vivos puedan desarrollarse y auto-replicarse (Karaguilla \& de Deus, 2008; Lima, Pereira, Oliveira, \& Werner, 2016; García-Peñalvo, Hernández-García, Conde, \& Fidalgo-Blanco, 2015).

Los ecosistemas digitales de aprendizaje representan un paradigma de los futuros sistemas de educación, soportados en las tecnologías de la información y las comunicaciones. Éstos se caracterizan por basarse en sistemas adaptativos capaces de modificarse a partir de diferentes relaciones o interacciones dadas en sentido simétrico entre los componentes del sistema: contexto, usuarios, contenidos, dispositivos, aplicaciones, formas de comunicación, entre otros (Motz \& Rodés, 2013; Barragán, 2016).

Con estos ecosistemas se busca enriquecer las experiencias de aprendizaje a través de actividades interactivas como pueden ser las simulaciones, juegos, etcétera; orientadas al modelo de comunidades de aprendizaje y al despliegue o exposición en soportes multidispositivo lo que enlaza con el desarrollo de soluciones m-learning (aprendizaje móvil) (García-Peñalvo \& Seoane-Pardo, 2015).

Por tanto, los elementos bióticos de un ecosistema digital corresponden al concepto de comunidades por ejemplo: redes sociales de contenidos específicos, desarrolladores de software libre, curadores de contenidos, comunidades de producción y exposición de contenidos. Por otra parte, los elementos abióticos serían concebidos como la infraestructura, la economía, la cultura y la legislación que sirven de soporte para la generación y evolución del ecosistema compuesto de espacios multidimensionales donde existen entidades que mutan, en este caso, los contenidos dirigidos a los individuos que se encargan de consumirlos o producirlos. El ambiente que circunda al ecosistema posee especies digitales identificadas como recursos o servicios web: blogs, wikis, vídeos en línea, repositorios, etcétera. Esta visión del ecosistema fortalece las características de adaptación y autorregulación donde los proveedores o consumidores pueden intercambiar roles.

Al mismo tiempo se habla del término ecología del aprendizaje (Añazco-Ojeda, sf; Coll, 2013), concebido desde los marcos teóricos que lo soportan, tales como la perspectiva ecológica que marca la relación entre el sujeto y el contexto como impulsor de los cambios del desarrollo en el que el individuo es quien configura su propio progreso, así, como la perspectiva histórico cultural, donde los elementos culturales aparecen mediando la actividad psicológica y las prácticas sociales que implican un cambio radical en los parámetros que intervienen en el aprendizaje: dónde se aprende, cuándo, cómo, con quién y de quién y sobre todo qué y para qué aprendemos. 
En este sentido, el concepto de ecología del aprendizaje se refiere al conjunto de contextos de actividad físicos o virtuales que proporcionan una variedad de posibilidades para generar aprendizajes que se reflejen en las prácticas realizadas por los sujetos cuando implican el uso de recursos, relaciones, destrezas y exigencias (AñazcoOjeda, sf).

Lo antes descrito también tiene implicaciones pedagógicas, en la ecología del aprendizaje las actividades surgen a espontaneidad durante el transcurso de un proceso formativo debido a las interacciones que ocurren entre estudiantes-tutores, estudiantescontenidos, estudiantes-tecnología, tutores-tecnología y es justamente esta interactividad lo que causa la evolución de los paradigmas pedagógicos siempre y cuando se visualicen desde la perspectiva de comunidad y no de individuos (Motz \& Rodés, 2013).

La ecología del aprendizaje deja entrever los retos que se plantean a la educación escolar y formal y las transformaciones que es necesario atender, dando paso a la personalización del aprendizaje entendida como la diversificación de las oportunidades, experiencias y recursos en función de las necesidades e intereses de los aprendices.

En la figura 1 se muestra una interpretación gráfica de lo que constituye un ecosistema de aprendizaje; como puede apreciarse hay un individuo que desarrolla habilidades y está en constante interacción con su contexto y las actividades que le permite desenvolverse en el ecosistema tanto en el aprendizaje informal como el formal, todo esto apoyado en las tecnologías digitales representadas por dispositivos, aplicaciones, herramientas, etcétera. Lo anterior ayudaría a garantizar un aprendizaje para la vida.

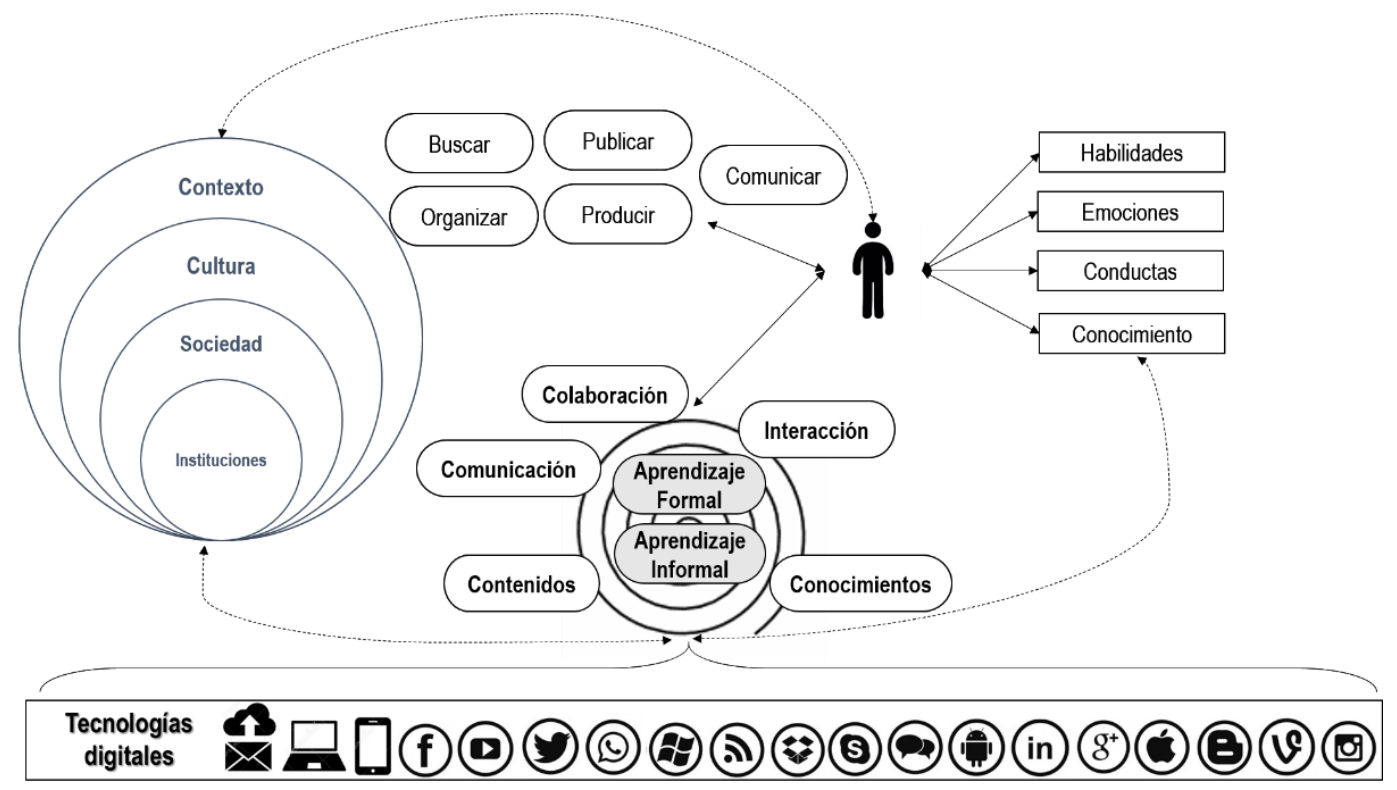

Figura 1. Ecosistema digital y su manifestación en el aprendizaje. Fuente: creación propia

\subsection{Manifestación de los ecosistemas digitales en el aprendizaje}


La manifestación de los ecosistemas digitales en el aprendizaje de los individuos parte del supuesto de que las herramientas, internet y sus aplicaciones, y los dispositivos electrónicos en conjunto ofrecen la posibilidad de que los individuos elijan sobre lo que aprenden, cuándo y cómo lo aprenden en función de sus tiempos y ritmos; permitiéndoles eliminar las posibles barreras de aprendizaje a las que pudieran enfrentarse.

Así pues las manifestaciones pueden observarse desde:

- La manera en que las tecnologías digitales modelan los escenarios de enseñanzaaprendizaje formales e informales, con la presencia de TIC con conexión inalámbrica o móviles ubicuos que permiten que el aprendizaje se desligue de los entornos físicos e institucionales.

- Las experiencias de aprendizaje que están modeladas por múltiples lenguajes y formas de expresión, además de sistemas multimedia y símbolos que hacen que la experiencia de aprender sea mayormente mediada por distintos vehículos y soportes.

- En los ecosistemas digitales el aprendizaje se orienta a la adquisición y dominio de habilidades y competencias genéricas o transversales que ayudan a seguir aprendiendo a lo largo de la vida (Coll, 2013).

- Las experiencias de aprendizaje que se mueven en función de los contextos por los que transitan los individuos, generándose rutas de aprendizaje personalizado donde el ecosistema les provee de oportunidades, recursos e instrumentos para aprender.

- Las tecnologías móviles e inalámbricas que permiten visualizar un modelo de aprendizaje centrado en que el estudiante aprenda lo que le interesa aprender en diferentes momentos y contextos de actividad (Reyna, 2011).

- Posibilitar a los estudiantes para que signifiquen lo aprendido independientemente del contexto en el que aprendan.

- La elección de lo que se aprende, cuándo y cómo se aprende ajustando el ritmo, la metodología y la inclusión eliminando barreras de aprendizaje.

- La generación de contextos de actividad por ejemplo: redes sociales, entornos virtuales, comunidades de interés, juegos en línea, que potencian el aprendizaje dentro y fuera de las instituciones de educación formal.

- La exposición de recursos o instrumentos que permiten aprender y que están a disposición de los estudiantes en distintos contextos de actividad.

- Generación de nuevas escenografías de comunicación para el aprendizaje donde interaccionen los alumnos y los profesores en prácticas innovadoras de enseñanza.

- El valor que se le da a la enseñanza destacando su visión transmisora y creadora sustentada en elementos epistémicos que respaldan la formación y el perfeccionamiento del profesorado.

- La movilización de competencias en experiencias innovadoras que buscan la aplicación de nuevos escenarios educativos donde los estudiantes no usan las TIC solo como herramientas tecnológicas e instrumentales.

- La colaboración para la construcción del conocimiento en comunidades donde la interacción fortalece la formación.

- El uso de las tecnologías para ayudar a explorar el conocimiento a través de la búsqueda, clasificación, interpretación y exposición de contenidos. 
- Los cambios pedagógicos que se suscitan en el aula y ambientes de aprendizaje donde se favorezca una enseñanza activa, participativa, colaborativa $y$ constructiva que guíe al estudiante a ser un ente activo de su proceso de enseñanza-aprendizaje.

- En la evolución de paradigmas pedagógicos donde la colaboración y aprendizaje se da a través de comunidades que intercambian intereses comunes.

- En la posibilidad que brindan los dispositivos para acceder a materiales didácticos promoviendo interacción entre docentes y estudiantes, cuidando el tratamiento y análisis de la información.

- La adquisición de conocimientos apoyándose en herramientas y pedagogías adecuadas.

- La intercomunicación y perfeccionamiento que busca un individuo en su proceso de formación a través de la instrucción, personalización y socialización.

- Percepciones favorables sobre el nivel de competencias mediáticas que se desarrollan de manera transversal en modelos de formación ecosistémica (Álvarez-Arregui, Rodríguez-Martín, Madrigal-Maldonado, \& GrossiSampedro, 2017).

A partir de lo anterior se infiere que las manifestaciones de los ecosistemas en el aprendizaje son múltiples y atienden a diferentes factores y elementos que van desde aspectos cognitivos, destrezas, formas de comunicación, cambios de paradigmas pedagógicos, entre otros; sin embargo, cabe destacar que en su mayoría la literatura encontrada carece de sustento empírico y datos duros que corroboren dichas manifestaciones, por lo que se quedan en el discurso y no en hechos que puedan constatarse a partir de datos cuantitativos o cualitativos como lo hacen Álvarez-Arregui, Rodríguez-Martín, Madrigal-Maldonado, \& Grossi-Sampedro (2017) .

\subsection{Los ecosistemas en la sociedad del conocimiento}

Inicialmente cabe aclarar que la sociedad del conocimiento es concebida como aquella en la que todos los miembros que la componen poseen capacidades y competencias para ser miembros activos de la construcción social del conocimiento (Barroso-Jerez, 2013).

Por tanto, los seres humanos integrados a una sociedad aprenden no sólo en las instituciones educativas sino a través de una serie de espacios, entornos y aplicaciones donde los sujetos interactúan con múltiples estímulos intercambiando información multi-sensorial y multicanal (Sevillano \& Vázquez, 2013) que altera significativamente los principios del fenómeno educativo, demandando formas renovadas de fundamentar el conocimiento y los procesos que facilitan su adquisición.

En este sentido, la sociedad del conocimiento se configura como un nuevo escenario donde sus elementos clave son la penetración de tecnologías en todos los sectores desde el industrial hasta el educativo, implicando un nuevo tipo de alumnos que al nacer en la generación .net tienen características cognitivas distintas a las de sus profesores o estudiantes de hace algunas décadas.

La sociedad en que nos desarrollamos actualmente está marcada por características tales como: tendencia hacia la globalización, girar en torno a las TIC, transformación de los conceptos de espacio y tiempo, amplitud y rapidez con que se genera la información y 
se expone ante los usuarios, generando una dinámica de aprender a aprender en una revolucionada sociedad en redes, alimentada por el conocimiento y contenidos producidos por los integrantes de la red (Cabero \& Marín, 2017). Es así, que las necesidades de comunicación y la búsqueda de alternativas para la interacción que ha implicado la penetración de las TIC ha generado una cultura mediática donde los usuarios tienen una nueva manera de crear símbolos, interpretaciones $\mathrm{y}$ reinterpretaciones sociales.

Por lo anterior, en la sociedad actual el abanico de posibilidades que representan los ecosistemas digitales ha dado paso a un capital humano distinto al de generaciones pasadas, exigiendo apropiarse de la modernidad de forma descentralizada, dando paso a nuevas competencias para interrelacionarse y modificar las actitudes y perspectivas de esas relaciones y su desarrollo como personas (Ibarra \& Llata, 2010).

Las características mencionadas han transformado la educación y los procesos de aprendizaje en una serie de direcciones tales como:

- Transformación y velocidad de cambio.

- Las instancias educativas normadas dejaran de ser las únicas instancias de formación.

- Transformación de las concepciones del aprendizaje en términos de colaboración y distribución.

- Entornos altamente tecnificados.

- La articulación del aprendizaje en función de lo síncrono y asíncrono.

- Formación del estudiante en nuevas competencias y capacidades.

- Cambios en las estructuras organizativas.

- La necesidad de configurar redes de información.

- Movilidad virtual del estudiante.

- Nuevos roles del profesor (Cabero \& Marín, 2017; Cáceres, Brändle, \& Ruíz, 2017) que adquieren significado a partir de las transformaciones en las concepciones del aprendizaje y en la modificación de los centros educativos en los que se acepta a las TIC para ponerlas a disposición de estudiantes y profesores.

Tras estos cambios, la ecología del aprendizaje implica para la sociedad del conocimiento un modelo de educación distribuido e interconectado que se adelante a la escolarización universal dando cabida a una multiplicidad de escenarios educativos, en los que participan distintos agentes atendiendo al dónde y con quién se aprende; asimismo, prolongar el aprendizaje a lo largo de la vida para tener una formación plena que lleve a los individuos a enfrentarse a situaciones cotidianas en las que apliquen las competencias o habilidades básicas requeridas para el siglo XXI.

De esta manera, los sujetos estarán preparados para seguir aprendiendo participando en comunidades de prácticas e intereses comunes, a través de múltiples formatos de representación de la información que principalmente estarán disponibles desde las TIC como vías de acceso a la información y el conocimiento (Coll, 2016) donde éstos fluyen y se reproducen para convertirse en elementos cada vez más esenciales y abundantes. 
En este mismo orden de ideas, los ecosistemas digitales se hacen presentes toda vez que los entes que lo constituyen posibilitan una realidad compartida en la que circula el conocimiento desde distintos canales o entornos físicos, además de las interacciones que se producen entre los individuos que componen la sociedad y cuyo desarrollo se sustenta en la construcción compartida de significados. Es así que puede afirmarse que el conocimiento en la sociedad evoluciona en interacción con el entorno sociocultural en que se desenvuelve (Barroso-Jerez, 2013); tal es el caso de los ecosistemas de ámbito territorial o de una comunidad determinada que se conectan en red para poner al alcance de sus habitantes las oportunidades, recursos e instrumentos para aprender de lo que disponen (Coll, 2016).

La especial influencia de las TIC representada por las redes sociales y la web 2.0 condujeron a un excedente cognitivo y de participación social donde las ganas de conocer, de participar, de aportar fueron más notorias (Reig, 2010), y la comunicación se hizo masiva originando nuevas redistribuciones de poder y de participación que en ocasiones reflejaron auto-organización de grupos, comunidades, nubes distribuidas donde la organización existe sin ser una entidad definida, sin un entorno físico que los sujete a algo institucional (Cáceres, Brändle, \& Ruíz, 2017).

Dichas transformaciones de concepción y organización ponen de manifiesto la necesidad de seguimiento y atención que en la sociedad del conocimiento debe darse a quienes han integrado las TIC en su cotidianeidad, para quienes la conexión ininterrumpida y ubicua se ha convertido en una forma de vida que inclusive llega a ser compulsiva y hasta adictiva, desconociendo donde están los límites de lo online y offline, ya que son pocos los espacios sociales y de conocimiento que no hayan sido transgredidos por la presencia de las tecnologías, cambiando las condiciones de los procesos y contextos de los sujetos así como sus prácticas y desempeños.

\section{Conclusiones}

Los efectos de la globalización cultural y económica, la ubicuidad de las tecnologías y el creciente lenguaje de símbolos y audiovisual que se genera reclama a los sistemas educativos que los estudiantes adquieran competencias suficientes para desempeñarse en un mundo que gira en torno a las TIC, y cuya velocidad y ritmo aún no ha sido alcanzado por las instituciones de educación.

Como se ha mencionado en las secciones anteriores, los nuevos escenarios, posibilidades de conexión, comunicación y socialización han generado desdibujamientos en la educación escolar; la cognición, capacidades y destrezas dejan de ser lineales, implicando demandas de formación que escapan a los entornos cerrados o áulicos, llevando al aprendizaje a expandirse a lo largo de la vida, requiriendo de los individuos competencias de responsabilidad, aprendizaje autogestivo, selección y discriminación de información, de manera que puedan generar sus propios entornos de aprendizaje de acuerdo a sus intereses y necesidades compartidas a nivel de comunidad.

En los ecosistemas las interacciones ya no se dan únicamente entre comunidades sino los factores contextuales, culturales y tecnológicos se suman con un efecto de cascada para los componentes del ecosistema. 
Como implicación pedagógica, la presencia de los ecosistemas digitales en el aprendizaje rompe los esquemas y estructuras de antaño, dando paso a la independencia y autonomía de los estudiantes siguiendo sus intereses y las situaciones que son significativas para ellos, interactuando además con otros estudiantes comportándose como sus formadores pares o participando en las comunidades.

Ante estos supuestos y discurso, no queda más que hacer mención sobre la importancia que tiene llevar a cabo investigaciones en el marco de los ecosistemas digitales y su manifestación en el aprendizaje, ya que son muy pocos los textos que se han encontrado cuyo fundamento sean datos empíricos cuantitativos o cualitativos que lleven a la medida o valoración de los ecosistemas.

Es importante saber hasta dónde este tipo de ecosistemas llegarán a su etapa de madurez y cómo se vigilará la diversidad que se produce al interior de éstos, en beneficio del aprendizaje y desarrollo de las comunidades que se les vinculan.

Como futuras líneas de actuación quienes aquí escriben se han propuesto realizar estudios empíricos en los que se dé cuenta de la incidencia de los ecosistemas digitales en el aprendizaje de los individuos. Resulta interesante resaltar que estos estudios también podrían dilucidar si el desarrollo de competencias digitales y su adecuada aplicación como mediación en el aprendizaje están asociadas a la existencia de los ecosistemas.

El camino por recorrer aún es largo y escabroso, al ser una temática no ahondada lo suficiente para esclarecer desde otra perspectiva la incidencia de las tecnologías de la información y comunicación en el contexto educativo.

Presentación del artículo: 12 de octubre de 2017 Fecha de aprobación: 28 de noviembre de 2017

Fecha de publicación: 22 de diciembre de 2017

Islas, C. y Carranza, M. (2017). Ecosistemas digitales y su manifestación en el aprendizaje: ¿Qué se dice al respecto? RED. Revista de Educación a Distancia, 55. Recuperado de: http://www.um.es/ead/red/55/islas_carranza.pdf

\section{Financiación}

Esta investigación no ha recibido ninguna subvención específica de los organismos de financiación en los sectores públicos, comerciales o sin fines de lucro.

\section{Referencias bibliográficas}

Álvarez-Arregui, E., Rodríguez Martín, A., Madrigal-Maldonado, R., \& GrossiSampedro, B. (2017). Ecosistemas de formación y competencia mediática: 
Valoración internacional sobre su implementación en educación superior. Comunicar, $X X V(51)$.

Añazco-Ojeda, D. (sf). Retos de la nueva ecología del aprendizaje: Personalización e intereses. Academia Edu. Recuperado de https://www.academia.edu/27220413/Retos_de_la_nueva_ecolog\%C3\%ADa_de 1_aprendizaje_Personalizaci\%C3\%B3n_e_intereses

Attwell, G. (2013). ¿Dónde vamos con los entornos personales de aprendizaje? En L. Castañeda , y J. Adell, Entornos personales de aprendizaje: Claves para el ecosistema educativo en red (págs. 189-192). Marfil.

Barragán, E. E. (2016). SlideShare. Recuperado de https://es.slideshare.net/elvesbarragan/ecosistemas-digitales-63429851

Barroso-Jerez, C. (2013). Sociedad del conocimiento y entorno digital. Teoría de la educación. Educación y cultura en la sociedad de la Información, 14(3), 61-86.

Bencini, P. G. (09 de 2000). Construir competencias. Periódico de la Universidad de Ginebra, págs. 19-31.

Cabero, J. (2015). Reflexiones educativas sobre las tecnologías de la información y la comunicación TIC. CEF(1), 19-27. Recuperado de www.tecnología-cienciaeducacion.com

Cabero, J., \& Marín, V. (2017). La educación formal de los formadores de la era digital - los educadores del siglo XXI. Notandum. doi:http://dx.doi.org/10.4025/notandum.44.4

Cáceres, M. D., Brändle, G., \& Ruíz, J. A. (2017). Sociabilidad virtual: la interacción social en el ecosistema digital. Historia y comunidad social, 22(1), 233-247.

Choque, R. (2009). Ecosistema educativo y fracaso escolar. Revista Iberoamericana de Educación, 4-10.

Coll, C. (2013). La educación formal en la nueva ecología del aprendizaje: tendencias, retos y agenda de investigación. Aprendizaje y educación en la sociedad digital. Barcelona: Universitat de Barcelona.

Coll, C. (2016). La personalización del aprendizaje escolar. El qué, el por qué, y el cómo de un reto insoslayable. Barcelona: Fundació Jaume Bofill.

García, M. L., \& Vázquez, E. (2013). La universidad ante el reto del aprendizaje ubicuo con dispositivos móviles. EDETANIA(44), 33-45.

García-Peñalvo, F. J. (2016). En clave de innovación educativa. Construyendo el nuevo ecosistema de aprendizaje. I Congreso Internacional de Tendencias en Innovación. Arequipa, Perú. Recuperado de https://repositorio.grial.eu/handle/grial/689 
García-Peñalvo, F., \& Seoane-Pardo, A. M. (2015). Una revisión actualizada del concepto de e-learning. Décimo aniversario. EKS, 16(1). doi:http://dx.doi.org/10.14201/eks2015161119144

García-Peñalvo, F., Hernández-García, Á., Conde, M. A., \& Fidalgo-Blanco, Á. (2015). Mirando hacia el futuro: Ecosistemas tecnológicos de aprendizaje basados en servicios. III Congreso Internacional sobre Aprendizaje, Innovación y Competitividad (CINAIC), (págs. 553-558). Madrid.

Ibarra, M. A., \& Llata, D. (2010). Niños nativos digitales en la sociedad del conocimiento: acercamientos conceptuales a sus competencias. Razón y Palabra, 15(72).

Karaguilla, I., \& de Deus, R. (2008). Digital Learning Ecosystems: Authoring, Collaboration, Inmersion and Mobility. IDC 2008 Proceedings (págs. 9-12). Chicago: ACM.

Lima, T., Pereira, R., Oliveira, J., \& Werner, C. (2016). The importance of socialtechnical resources for software ecosystems management. Journal of innovation in digital ecosystems(3), 98-113.

Motz, R., \& Rodés, V. (2013). www.laclo.org. Recuperado de www.laclo.org/papers/index.php/laclo/article/download/99/92

Reig, D. (2010). El futuro de la educación superior, algunas claves. REIRE, 3(2), 98113.

Reyna, J. (2011). Digital Teaching and Learning Ecosystem (DTLE): a Theorical Approach for Online Learning Environments. Ascilite 2011 changing demands, changing directions, (págs. 1083-1088). Hobart Tasmania. Recuperado de http://www.ascilite.org/conferences/hobart11/downloads/papers/Reynaconcise.pdf

Sevillano, M. L., \& Vázquez, E. (2013). La universidad ante el reto del aprendizaje ubicuo con dispositivos móviles. EDETANIA(44), 33-45. 